Revista de Psicología y Educación / Journal of Psychology and Education, 2018, 13(2), 99-1 12 (www.rpye.es) Doi: https://doi.org/10.23923/rpye2018.01.161

ISSN: $1699-9517$

\title{
Resiliencia, bienestar psicológico y afrontamiento en universitarios atendiendo a variables de personalidad y enfermedad María Hinojal Benavente-Cuesta* y María Paz Quevedo-Aguado
}

Facultad de Ciencias de la Salud y Facultad de Psicología. Universidad Pontificia de Salamanca

Resumen: Existen investigaciones que desde perspectivas diferentes han tratado de aproximarse a constructos psicológicos como la Resiliencia, el Bienestar Psicológico, las Estrategias de Afrontamiento y la Personalidad. Este estudio se centra en la población universitaria, analizando cada uno de estos constructos y las posibles relaciones entre ellos. Participaron 447 estudiantes con una media de edad de 21.11 años. Entre las variables más destacadas para su análisis, a parte de las sociodemográficas, se encontraban el tipo de titulación que cursaban y la presencia o no de algún tipo de enfermedad crónica. Se estudiaron las posibles diferencias al comparar los subgrupos de la muestra determinados por la edad, sexo, titulación y presencia de enfermedad. Instrumentos utilizados: Cuestionario de Resiliencia de Wagnild y Young; Escala de Bienestar Psicológico de Ryff; Minimarcadores de los Cinco Grandes en Español; Cuestionario de Afrontamiento del Estrés y Cuestionario de Percepción de enfermedad. Los resultados indicaron una relación entre la Resiliencia y el Bienestar Psicológico, así como con la dimensión de personalidad Estabilidad emocional y con dos de las Estrategias de afrontamiento.Se hallaron diferencias en algunas de las dimensiones de estos constructos al comparar grupos según edad, sexo y tipo de titulación, no asi con la presencia de enfermedad.

Palabras clave: Resiliencia, Bienestar Psicológico, Afrontamiento, Personalidad y Percepción enfermedad.

\section{Resilience, psychological well-being and university collaboration attending variables of personality and disease}

Abstract: There are researches that from different perspectives have tried to approach psychological constructs such as Resilience, Psychological Wellbeing, Coping Strategies and Personality. This study focuses on the university population, analyzing each of these constructs and the possible relationships between them. Participants were 447 students with an average age of 21.11 years. Among the most important variables for analysis, apart from the sociodemographic ones, were the type of degree they were studying and the presence or not of some type of chronic disease. The possible differences were studied when comparing the subgroups of the sample determined by age, sex, degree and presence of disease. Instruments used: Wagnild and Young Resilience Questionnaire; Ryff Psychological Well-Being Scale; Minimizers of the Big Five in Spanish; Stress Coping Questionnaire and Disease Perception Questionnaire. The results indicated a relationship between Resilience and Psychological Wellbeing, as well as with the personality dimension Emotional Stability and with two of the Coping Strategies. Differences were found in some of the dimensions of these constructs when comparing groups according to age, sex and gender. type of degree, not so with the presence of disease.

Keywords: Resiliencie, Psychological Well-being, Coping, Personality and Perception disease.

La Resiliencia se viene estudiando desde las últimas décadas del siglo XX (Becoña, 2006), siendo especialmente objetos de estudio la población infantil y adulta con algún tipo

Recibido: 27/11/2017 - Aceptado: 17/1/2018 - Avance online: 06/3/2018 *Correspondencia: María Hinojal Benavente Cuesta.

Universidad Pontificia de Salamanca.

C. P: 37002, Salamanca, España.

E-mail: mhbenaventecu@upsa.es de patología crónica (Büssing, Ostermann y Koenig, 2007). Son muchas las definiciones que se han ido proponiendo, ya que es un constructo aún en un continuo proceso de evolución y maduración a expensas de los nuevos resultados arrojados por la investigación. Existe, sin embargo, consenso en afirmar, que se trata de un proceso dinámico, que cambia 
en el transcurso de la vida; una capacidad humana para enfrentar, sobreponerse y salir fortalecido en contextos de adversidad, eventos o experiencias estresantes (Becoña, 2006; Luthar, 2006). En este proceso interaccionan dos tipos de factores: protectores y de riesgo. Los factores protectores o también llamados amortiguadores del estrés, hacen referencia a las influencias que modifican, mejoran o alteran la respuesta de una persona a algún peligro que predispone a un resultado no adaptativo (Rutter, 1985, 1987). Reducen los efectos negativos de la exposición a factores de riesgo y al estrés, de modo que algunas personas llevan una vida normal a pesar de vivir en contextos con situaciones desfavorables (Bernard, 2004; Rutter, 2007). Entre ellos se distinguen aquellos que pertenecen a las características ambientales que rodean al individuo, y aquellos que se deben a las cualidades y habilidades individuales (Wolin y Wolin, 1993). Por otro lado, los factores de riesgo representan variables que ante situaciones adversas incrementan la posibilidad de respuestas negativas aumentando así la vulnerabilidad de las personas, no desencadenan siempre un enlace negativo, pero su presencia potencia la probabilidad de ocurrencia (Bernard, 2004).

Junto a la Resiliencia, se estudiará el Bienestar psicológico, otro gran constructo al que le avala una amplía literatura científica. $\mathrm{Ha}$ sido estudiado desde diferentes enfoques pero en esta investigación se asumirá el Modelo multidimensional sugerido porRyff(1989), para el cual, el Bienestar psicológico está constituido por seis dimensiones: Autoaceptación, Relaciones positivas con otras personas, Autonomía, Dominio del entorno, Propósito en la vida y Crecimiento personal. Estudiar la relación entre la Resiliencia y el Bienestar psicológico podrá ayudar a entender y comprender mejor lo que supone el funcionamiento óptimo de la persona cualquiera que sea la situación favorable o no, poero manteniendo un cierto grado de bienestar. Este modo particular de funcionamiento implica, saber afrontar y gestionar situaciones de estrés. Para analizar esta cuestión, se tomará como referencia la perspectiva propuesta por Lazarus y Folkman (1986) que concibe el afrontamiento como proceso, ya que esta es la que ha adquirido mayor desarrollo en los últimos años. Se define, como los esfuerzos cognitivos y conductuales dirigidos a manejar las demandas internas o externas evaluadas como algo que agrava o excede los recursos de la persona. Esta concepción asume que el individuo utiliza diversas estrategias para tratar con el estrés, las cuáles pueden ser o no adaptativas. La cuestión que nos interesa en este trabajo, es conocer la vinculación entre las Estrategias de Afrontamiento con la Resiliencia y el Binestar psicológico. ¿̇Las personas más resilientes y/o las personas que gozan de un cierto grado de bienestar psicológico son más procribles a desarrollar un determinado tipo de estrategias?

Así mismo, se cuestionará si existe algún tipo de relación entre Personalidad, Resiliencia, Bienestar psicológico y Afrontamiento. Se aceptará el concepto de personalidad propuesto por el Modelo de los Cinco Grandes (Goldberg, 1981), modelo psicoléxico que considera que existen cinco grandes dimensiones de personalidad, constituyendo éstas los pilares básicos que captan la esencia de las diferencias individuales: Extraversión, Afabilidad, Neuroticismo, Responsabilidad y Apertura a la experiencia. Dicho modelo cuenta con un alto grado de solidez empírica, demostrándose en diferentes tipos de estudios, la relación entre algunas de estas dimensiones con la Resiliencia y el Bienestar Psicológico (Friborg, Barlaug, Martinussen, Rosenvinge y Hjemdal, 2005; Kobasa, 1979; Vázquez, Hervás, Rahona y Gómez, 2009).

Por otro lado, señalar que en esta investigación una de las variables especialmente atendidas ha sido si la persona tenía o no algún tipo de enfermedad, siendo considerado este hecho como una situación estresante capaz de marcar diferencias respecto a aquellos que no viven esta realidad.

Par finalizar, teniendo en cuenta todo lo anteriormente expuesto, se señalan los principales objetivos que han guiado este estudio: Conocer la relación entre la Resiliencia, el Bienestar psicológico, las Estrategias de Afrontamiento y 
la Personalidad así como analizar las posibles diferencias existentes en estos constructos al comparar los diferentes grupos de la muestra en función de la edad, el sexo, tipo de titulación y si padecen o no algún tipo de enfermedad.

\section{MÉTODO}

\section{PARTICIPANTES}

Participaron 458 estudiantes de la Universidad Pontificia de Salamanca (España). El 60.3\% son de Enfermería, el 36.2\% de Psicología, el $1.1 \%$ pertenecen a grados no sanitarios y el 2,4\% profesionales.La media de edad es de 21.11 años (DT=3.9), siendo el mínimo 17 y el máximo 49 años. Las mujeres representan el $77.7 \%$ frente al $22.3 \%$ de hombres. El $55.9 \%$ están solteros, el $41.9 \%$ tienen pareja estable, el $1.3 \%$ casados y el $0.9 \%$ se encuentran en otros estados.Atendiendo a la variable enfermedad,el $81.2 \%$, no padecen ningún tipo de patología, mientras que el $18.8 \%$ se pueden considerar enfermos crónicos, al observar que, el $64.7 \%$ llevan más de 5 años con la enfermedad, el $27.1 \%$ entre uno y cinco años, y tan sólo el $8.2 \%$ menos de un año. Las enfermedades con mayor representación son: asma, migrañas, diabetes mellitus tipo 1, alergias, dermatitis, intolerancias a ciertos alimentos, epilepsia, TDAH e hipotiroidismo, ansiedad, depresión y estrés.

\section{INSTRUMENTOS}

Datos Sociodemográficos: Edad, Sexo, Estado civil, Titulación y Curso

Cuestionario de Resilienciade Wagnild y Young (1993). Adaptado a la versión española por Heilemann, Lee y Kury (2003). Escala tipo likert formada por 25 ítems cuyas puntuaciones pueden ser: (1) En absoluto, (2) Rara vez, (3) $A$ veces (4) A menudo y (5) Casi siempre. La fiabilidad de la escala total es de $\alpha=$.92.Se distribuyen en dos dimensiones Competencia personal $(\alpha=.88)$ y Aceptación de uno mismo y de la vida $(\alpha=.81)$, a su vez se presentan 4 subdimensiones: Satisfacción personal,
Ecuanimidad, Sentirse bien solo y Confianza en sí mismo.

Escala de Bienestar Psicológico deRyff (1989). Versión reducida y adaptada al español por Díaz, Rodríguez, Blanco, Moreno, Gallardo, Valle y van Dierendonck, (2006). Consta de 29 ítems, valorados en una escala tipo Likert que va de (1) Nada adecuado, (2) Mínimamente adecuado, (3) Algo adecuado, (4) Bastante adecuado y (5) Muy adecuado. Se distribuye en 6 dimensiones: Autoaceptación $(\alpha=.84)$, Relaciones positivas con otras personas $(\alpha=.78)$, Autonomía $(\alpha=.70)$, Dominio del entorno $(\alpha=.82)$, Propósito en la vida $(\alpha=.70)$ y Crecimiento personal $(\alpha=.71)$.

Minimarcadores de los Cinco Grandes en Español (Iraegui y Quevedo-Aguado, 2002). Consta de 150 adjetivos identificados como descriptores de personalidad, aunque se ha utilizado una versión reducida de 50 ítems. Escala tipo Likert cuyas puntuaciones se distribuyen entre; (1) No adecuada, (2) Poco adecuada, (3) Adecuada (4) Moderadamente Adecuada y (5) Muy adecuada. La fiabilidad global del instrumento es de $\alpha=.884$, encontrándose cada una de los cinco factores en índices que oscilan entre $\alpha=.79$ y $\alpha=.89$; Fl Estabilidad Emocional (Ej: seguro, positivo, valiente), Fll Agradabilidad (Ej. apacible, aplicado,tolerante...)., FIII Integridad (Ej:profundo, receptivo, reflexivo), FIV Responsabilidad (Ej. responsable, constante, centrado) y FV Extraversión (Ej. abierto, alegre, comunicativo...).

Cuestionario de Afrontamiento del Estrés (CAE) (Sandin y Chorot, 2003). Escala tipo Likert cuyas puntuaciones se distribuyen entre: (1) Nunca, (2) Pocas veces, (3) A veces (4) Frecuentemente y (5) Casi siempre. La fiabilidad oscila entre $\alpha=.64$ y $\alpha=.92$ en los 7 factores: Focalización en la solución de problemas, Autofocalización negativa, Reevaluación positiva, Expresión Emocional Abierta, Evitación, Búsqueda de apoyo social y Religión.

Cuestionario de Percepción de enfermedad (Broadbent, Petrie, Main yWeinman, 2006). 
Escala tipo Likert(0-10) que evalúa la percepción de enfermedad, atendiendo a; consecuencias (Ej.Cuánto afecta su enfermedad a su vida), duración, control personal (Ej. Cuánto control cree Ud. que tiene sobre su enfermedad), tratamiento, identidad, preocupación ( $\mathrm{E}_{\mathrm{j}}$. En qué medida está Ud. preocupado por su enfermedad), respuesta emocional y comprensión de la propia enfermedad.Tiene un bajo índice de fiabilidad $\alpha=.44$, por lo que debería ser revisado, pudiendo deberse a la longitud de la escala, o al carácter clínico con el que fue construido.

\section{PROCEDIMIENTO}

En el curso 2016-2017,los investigadores de este estudio aplicaron a estudiantes matriculados en Enfermería, Psicología y otros Grados no sanitarios de la UPSA, los cuestionarios descritos, en formato papel. Contestados individualmente de forma voluntaria durante aproximadamente 30 minutos en horas lectivas de clase, asegurándoles el anonimato y la confidencialidad de los datos.

\section{ANÁLISIS DE DATOS}

Los datos obtenidos a través de las diferentes escalas utilizadas han sido tratados con el paquete estadístico SPSS. 15.0. Se presentarán los análisis que han resultado significativos.

\section{RESULTADOS}

Relación entre Resiliencia, Bienestar Psicológico, Estrategias de Afrontamiento y Personalidad.

Aparecen un elevado número de correlaciones según el coeficiente de Pearson. Se presentan en la Tabla 1, aquellas que han resultado significativas a un nivel de 0.01 (bilateral).

La Resiliencia total, muestra relaciones positivas con: Propósito en la vida (.72), Autoaceptación (.67), Dominio del Entorno (.53), Crecimiento Personal (.52), y Autonomía (.42). A medida que el sujeto es más resiliente aumentan cada una de estas dimensiones de bienestar, y a la inversa.

\begin{tabular}{|c|c|c|c|c|c|c|c|c|c|c|c|}
\hline \multicolumn{12}{|c|}{$\begin{array}{c}\text { Tabla } 1 \\
\text { Correlaciones de Pearson entre Resiliencia, Bienestar Psicológico, Estrategias de Afrontamiento y Personalidad }\end{array}$} \\
\hline & $\begin{array}{l}: 0 \\
: 0 \\
0 \\
\frac{0}{0} \\
0 \\
0 \\
0 \\
\frac{0}{3} \\
\frac{1}{2}\end{array}$ & $\begin{array}{l}\frac{0}{\varepsilon} \\
\frac{0}{c} \\
\frac{0}{3} \\
\frac{0}{4}\end{array}$ & 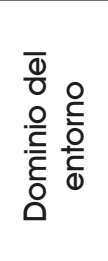 & 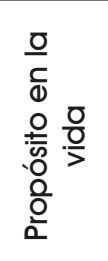 & 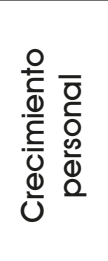 & 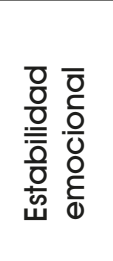 & 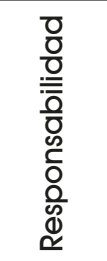 & 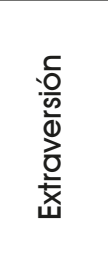 & 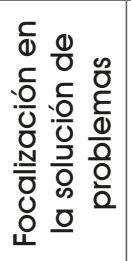 & 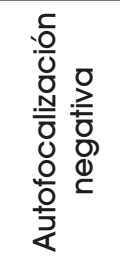 & 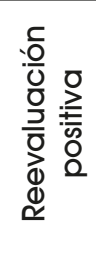 \\
\hline Resiliencia & $\left..677^{* *}\right)$ & $.42(* \star)$ & $.53(* \star)$ & $.72(* \star)$ & $.52(* \star)$ & $.60(* *)$ & $.38\left(^{* \star}\right)$ & $.38\left(^{* \star}\right)$ & $.51(* \star)$ & $-.42(* *)$ & $.47\left(^{* *}\right)$ \\
\hline $\begin{array}{l}\text { Satisfacción } \\
\text { Personal }\end{array}$ & $.59(* *)$ & & $.51(* *)$ & $.70(* \star)$ & $\left..400^{* *}\right)$ & $\left..488^{\star \star}\right)$ & $\left..377^{* *}\right)$ & & $\left..411^{* *}\right)$ & $-.37(* *)$ & \\
\hline Ecuanimidad & $.57\left(^{* *}\right)$ & $.36\left(^{* \star}\right)$ & $.45(* *)$ & $.58\left(^{\star \star}\right)$ & $\left..399^{* *}\right)$ & $.47(\star \star)$ & & & $.42(* *)$ & & $.36\left(^{* *}\right)$ \\
\hline Sentirse bien solo & $.37\left(^{* \star}\right)$ & & & $.40(* \star)$ & $.35\left(^{\star \star}\right)$ & & & & & & $.35\left(^{\star \star}\right)$ \\
\hline $\begin{array}{l}\text { Confianza en sí } \\
\text { mismo }\end{array}$ & $.54(* \star)$ & $.36\left(^{* *}\right)$ & $.43(* \star)$ & $.63(* *)$ & $\left..49^{\star \star}\right)$ & $.52(* \star)$ & $\left..377^{* \star}\right)$ & $\left..39{ }^{* \star}\right)$ & $.42(* *)$ & & $.41(* *)$ \\
\hline Autoaceptación & & & & & & $.62(* \star)$ & & .35 & $.40(* \star)$ & $-.48\left(^{(\star *}\right)$ & $.43(* *)$ \\
\hline Autonomía & & & & & & $-45(* \star)$ & & & & $-.40\left(^{* \star}\right)$ & \\
\hline Dominio del entorno & & & & & & $.42(* *)$ & & & & & \\
\hline Propósito en la vida & & & & & & $.46\left(^{* *}\right)$ & $.43(* *)$ & & $\left..477^{* *}\right)$ & $-.38\left(^{(\star *}\right)$ & $.46\left(^{* *}\right)$ \\
\hline $\begin{array}{l}\text { Crecimiento } \\
\text { personal }\end{array}$ & & & & & & & & & & & $.42\left(^{* *}\right)$ \\
\hline $\begin{array}{l}\text { Autofocalización } \\
\text { negativa }\end{array}$ & & & & & & $\left.-.399^{* \star}\right)$ & & & & & \\
\hline
\end{tabular}


Respecto a las Estrategias de Afrontamiento, hallamos relaciones positivas entre la Resiliencia total y la Focalización en la solución de problemas (.51) y la Reevaluación Positiva (.47). Cuánto más aumentan los índices de resiliencia en el sujeto más se incrementan el uso de estas dos estrategias, y viceversa. De forma diferente, sucede con la Autofocalización Negativa, que mantiene una relación inversa (-.42). Cuánto más resiliente es el sujeto menos desarrolla esta estrategia de afrontamiento negativa, y a la inversa, a menor Resiliencia total mayor Autofocalización negativa.

En relación con la Personalidad, descubrimos una relación positiva con la dimensión positiva de: Estabilidad Emocional (.60), Responsabilidad (.38) y Extraversión (.38), e inversa, con la dimensión negativa del primer Factor (-.53). Parece indicar que la Resiliencia aumenta a medida que el sujeto presenta características de personalidad relacionadas con la estabilidad emocional, la responsabilidad y la extraversión, y viceversa.

En cuanto a las subdimensiones de la Resiliencia, hallamos que la Satisfacción personal, correlaciona de forma positiva con: Propósito en la vida (.70), Autoaceptación (.59), Dominio del entorno (.51) y Crecimiento Personal (.40). Esto indica la estrecha relación entre estas dimensiones, las cuáles giran en torno al concepto de satisfacción alcanzado por el sujeto al comprender su significado y contribución a la vida.Existe una correlación positiva con la Focalización en la solución de problemas (.41), y negativa con la Autofocalización negativa (-.37). Aparece también una relación positiva con la dimensión positiva del Factor Estabilidad Emocional (.48) y el Factor Responsabilidad (.37), pero sin embargo,surge una relación negativa con la dimensión negativa del Factor Estabilidad Emocional (-.46). A mayor estabilidad emocional y responsabilidad mayor satisfacción personal, por el contrario, a mayor inestabilidad emocional menor grado de satisfacción.

La Ecuanimidad, muestra una correlación positiva con: Propósito en la vida (.58), Autoaceptación (.57), Dominio del entorno (.45),
Crecimiento personal (.39) y Autonomía (.36). Respecto a las estrategias de Afrontamiento, surge una relación positiva con: Focalización en la solución de problemas (.42) y Reevaluación Positiva (.36). Una vez más, la Estabilidad Emocional, presenta una relación significativa en su dimensión positiva (.47) e inversa con la dimensión negativa dela misma (-.46).A medida que crece la posición equilibrada ante la vida aumentan las distintas dimensiones anteriormente descritas del Bienestar, y esto además guarda una relación directa con las características personales de estabilidad emocional.

Sentirse bien, es la que menos relaciones obtiene en comparación con el resto. Correlaciones positivas con: Propósito en la vida (.40), Autoaceptación (.37) y Crecimiento Personal (.35). El sentimiento de bienestar aumenta al mismo tiempo que se incrementa el significado y sentido de la vida, el crecimiento personal y la aceptación de uno mismo. Surge también otra relación de carácter positivo, entre Sentirse bien y la Reevaluación positiva (.35).

La Confianza en sí mismo, es la que presenta mayor número de relaciones significativas. Relaciones positivas con: Propósito en la vida (.63), Autoaceptación (.54), Crecimiento personal (.49), Dominio del entorno (.43) y Autonomía (.36). Parece demostrarse que al aumentar la confianza en uno mismo, las distintas dimensiones del Bienestar psicológico también aumentan, y al revés, al disminuir la confianza en uno mismo disminuyen dichas dimensiones. También, se observa relación positiva con: Focalización en la solución de problemas (.42) y Reevaluación positiva (.41). Respecto a la Personalidad, aparecen tres correlaciones positivas con las dimensiones positivas de: Estabilidad Emocional (.52), Extraversión (.39) y Responsabilidad (.37), y una negativa con la dimensión negativa de Responsabilidad (-.46).

La Perseverancia, de igual modo que en los casos anteriores, muestra relaciones positivas con: Propósito en la vida (.54), Autoaceptación (.56), Dominio del entorno (.45), Crecimiento personal (.42) y Autonomía (.41). Igualmente, con Focalización en la solución de problemas 
(.42) y Reevaluación Positiva (.38). Finalmente, ha presentado relaciones significativas con las dos dimensiones positiva (.52) y negativa (-.43) del Factor Estabilidad Emocional. Las personas con niveles altos de Perseverancia, muestran mayor persistencia ante la adversidad y tienen un fuerte deseo de logro y autodisciplina, al aumentar estas características crecen también las dimensiones del Bienestar psicológico antes señaladas. $Y$ una vez más, asume un gran protagonismo las formas de Afrontamiento centradas en la Solución de problemas y la Reevaluación positiva, así como la variable de personalidad Estabilidad Emocional.

Atendiendo a la dimensión del Bienestar psicológico, Autoaceptación, han aparecido relaciones significativas positivas con dos estrategias de afrontamiento: Reevaluación positiva (.43) y Focalización en la solución de problemas (.40), y una negativa con la Autofocalización negativa (-.48). Además, la Autoaceptación, tiene una relación positiva con las dimensiones positivas de personalidad, Estabilidad Emocional (.62) y Extraversión (.35), y negativa con la subdimensión negativa del factor Estabilidad Emocional (-.62).

Respecto a la Autonomía, surge una relación significativa negativa con la Autofocalización
Negativa (.40) y con las dos dimensiones positiva (.45) y negativa (-.48) de la Estabilidad Emocional, de tal modo, que al aumentar la Autonomía del sujeto se incrementa la Estabilidad Emocional. De la misma manera, sucede con Dominio del entorno, presenta una relación significativa con la Estabilidad Emocional, en su dimensión positiva (.42)y negativa (-.47).

El Crecimiento personal, correlaciona de forma positiva con, Focalización en la solución de problemas (.38) y Reevaluación positiva (.42).

Y por último, el Propósito en la vida, igual que en dimensiones anteriores, se relaciona de forma positiva con Focalización en la solución de problemas (.47) y Reevaluación positiva (.46) y negativa con la Autofocalización negativa (-.38), además con los Factores de personalidad Estabilidad Emocional en su dimensión positiva (.46) y negativa (-.44), y con el Factor Responsabilidad en su dimensión positiva (.43).

Para finalizar la Autofocalización negativa, presenta una relación significativa con la Estabilidad Emocional, en su dimensión positiva (-.39) y negativa (.49).

Tabla 2

ANOVA de la Resiliencia y Personalidad en función de la Titulación

\begin{tabular}{|c|c|c|c|c|c|c|}
\hline & & $\begin{array}{l}\text { Suma de } \\
\text { cuadrados }\end{array}$ & gl & $\begin{array}{c}\text { Media } \\
\text { cuadrática }\end{array}$ & $\mathrm{F}$ & Sig. \\
\hline \multirow{3}{*}{ Ecuanimidad } & Inter-grupos & 102,180 & 3 & 34,060 & 5,189 & ,002 \\
\hline & Intra-grupos & 2966,852 & 452 & 6,564 & & \\
\hline & Total & 3069,033 & 455 & & & \\
\hline \multirow{3}{*}{$\begin{array}{l}\text { Confianza en sí } \\
\text { mismo }\end{array}$} & Inter-grupos & 126,871 & 3 & 42,290 & 3,330 & , 020 \\
\hline & Intra-grupos & 5714,364 & 450 & 12,699 & & \\
\hline & Total & 5841,236 & 453 & & & \\
\hline \multirow{3}{*}{ Resiliencia } & Inter-grupos & 1158,941 & 3 & 386,314 & 2,820 & ,039 \\
\hline & Intra-grupos & 61095,504 & 446 & 136,985 & & \\
\hline & Total & 62254,444 & 449 & & & \\
\hline \multirow{3}{*}{$\begin{array}{c}\text { F3 Integridad } \\
(+)\end{array}$} & Inter-grupos & 4,427 & 3 & 1,476 & 4,352 & ,005 \\
\hline & Intra-grupos & 153,252 & 452 & ,339 & & \\
\hline & Total & 157,679 & 455 & & & \\
\hline \multirow{3}{*}{ F3 Integridad (-) } & Inter-grupos & 2,676 & 3 & ,892 & 3,704 & , 012 \\
\hline & Intra-grupos & 108,150 & 449 & ,241 & & \\
\hline & Total & 110,826 & 452 & & & \\
\hline
\end{tabular}


Diferencias en Resiliencia y Personalidad en función de la Titulación

Al comparar los grupos de estudiantes según la titulación aparecen diferencias en el nivel de Resiliencia total y en las subdimensiones: Ecuanimidad y Confianza en sí mimo, así como en el FIII de personalidad denominado Integridad (Tabla 2).
Respecto a la Ecuanimidad, capacidad del sujeto para asumir una perspectiva equilibrada y moderada ante la adversidad, encontramos que los estudiantes de Enfermería tienen una media significativamente mayor (prueba de Scheffé) (15.5) en comparación con los estudiantes de otras titulaciones (1 1.60) (Tabla 3). Éstos últimos muestran también diferencias con los estudiantes de psicología, cuya media

\begin{tabular}{|c|c|c|c|c|c|c|c|}
\hline \multicolumn{8}{|c|}{$\begin{array}{c}\text { Tabla } 3 \\
\text { Descriptivos Resiliencia y Personalidad en función de la Titulación }\end{array}$} \\
\hline & & \multirow[b]{2}{*}{$N$} & \multirow[b]{2}{*}{ Media } & \multirow[b]{2}{*}{$\begin{array}{l}\text { Desviación } \\
\text { típica }\end{array}$} & \multirow[b]{2}{*}{ Error típico } & \multicolumn{2}{|c|}{$\begin{array}{l}\text { Intervalo de confianza } \\
\text { para la media al } 95 \%\end{array}$} \\
\hline & & & & & & $\begin{array}{l}\text { Límite } \\
\text { inferior }\end{array}$ & $\begin{array}{l}\text { Límite } \\
\text { superior }\end{array}$ \\
\hline \multirow{5}{*}{ Ecuanimidad } & 1,00 & 274 & 15,51 & 2,549 & , 154 & 15,20 & 15,81 \\
\hline & 2,00 & 166 & 15,80 & 2,521 & , 196 & 15,41 & 16,19 \\
\hline & 3,00 & 5 & 11,60 & 4,722 & 2,112 & 5,74 & 17,46 \\
\hline & 4,00 & 11 & 14,45 & 2,339 & ,705 & 12,88 & 16,03 \\
\hline & Total & 456 & 15,55 & 2,597 & 122 & 15,31 & 15,79 \\
\hline \multirow{5}{*}{$\begin{array}{l}\text { Confianza en } \\
\text { sí mismo }\end{array}$} & 1,00 & 274 & 27,74 & 3,490 & ,211 & 27,32 & 28,15 \\
\hline & 2,00 & 164 & 27,47 & 3,648 & ,285 & 26,91 & 28,03 \\
\hline & 3,00 & 5 & 22,80 & 5,263 & 2,354 & 16,27 & 29,33 \\
\hline & 4,00 & 11 & 28,18 & 3,311 & ,998 & 25,96 & 30,41 \\
\hline & Total & 454 & 27,60 & 3,591 & , 169 & 27,27 & 27,93 \\
\hline \multirow{5}{*}{ Resiliencia } & 1,00 & 270 & 96,12 & 11,400 & ,694 & 94,76 & 97,49 \\
\hline & 2,00 & 164 & 95,59 & 12,073 & ,943 & 93,73 & 97,45 \\
\hline & 3,00 & 5 & 81,00 & 17,378 & 7,772 & 59,42 & 102,58 \\
\hline & 4,00 & 11 & 94,09 & 10,821 & 3,263 & 86,82 & 101,36 \\
\hline & Total & 450 & 95,71 & 11,775 &, 555 & 94,62 & 96,80 \\
\hline \multirow{5}{*}{$\begin{array}{l}\text { FIII Integridad } \\
\qquad(+)\end{array}$} & 1,00 & 275 & 3,68 &, 579 & ,035 & 3,61 & 3,75 \\
\hline & 2,00 & 165 & 3,86 & ,577 & ,045 & 3,77 & 3,94 \\
\hline & 3,00 & 5 & 3,36 &, 974 & ,435 & 2,15 & 4,57 \\
\hline & 4,00 & 11 & 3,55 & ,552 & , 166 & 3,17 & 3,92 \\
\hline & Total & 456 & 3,74 & ,589 & ,028 & 3,68 & 3,79 \\
\hline \multirow{5}{*}{$\begin{array}{c}\text { FIII Integridad } \\
\qquad(-)\end{array}$} & 1,00 & 273 & 1,50 &, 503 & ,030 & 1,44 & 1,56 \\
\hline & 2,00 & 164 & 1,47 & ,466 & ,036 & 1,39 & 1,54 \\
\hline & 3,00 & 5 & 2,16 &, 767 & ,343 & 1,21 & 3,11 \\
\hline & 4,00 & 11 & 1,33 & ,393 & 118 & 1,06 & 1,59 \\
\hline & Total & 453 & 1,49 & 495 & ,023 & 1,45 & 1,54 \\
\hline
\end{tabular}


15.8, es mayor. No existen diferencias ente los estudiantes de Enfermería y Psicología. A la luz de estos resultados se puede deducir que los futuros enfermeros y psicólogos reflejan una mayor capacidad para mostrar actitudes equilibradas ante la adversidad en comparación con los estudiantes que no son de las ciencias de la salud.

En cuanto a la Confianza en sí mismo, encontramos resultados semejantes a los de la dimensión anterior: los alumnos de Enfermería (27.74) y Psicología (27.47), presentan una media superior en relación con otras titulaciones (22.80), lo que indica mayor habilidad para creer en ellos mismos y en sus capacidades, y de nuevo refleja la proximidad entre ambas muestras y la diferencias con otros estudiantes de ámbitos ajenos a la formación sanitaria.

En la Resiliencia Total, existen diferencias entre la titulación de Enfermería y las no sanitarias (otras tilulaciones). Al comparar las medias se observa que los primeros obtienen una mediade 96.12, dato muy superior al otro grupo que es de 81.00. Esto indica que los futuros enfermeros muestran una mayor capacidad de resistencia ante la adversidad en comparación con los otros estudiantes.

Por último señalar que, en referencia a las variables de Personalidad, tan sólo se han encontrado diferencias en el Factor Integridad.
Analizando las medias, los estudiantes de Psicología (3.86) se describen más receptivos, reflexivos, confiables y profundos que los de Enfermería (3.68). En relación a la dimensión negativa de este factor, también surgen discrepancias entre los distintos grupos. Los alumnos de Enfermería no se describen (1.50) como personas desleales, fanfarronas, troleras, malvadas e hipócritas, mientras que los pertenecientes a otras titulaciones (no en el caso psicología) esta puntuación se eleva de forma considerable (2.16). Lo mismo ocurre entre este último grupo y los estudiantes de Psicología (1.47).

Debemos entender estos resultados con un alto nivel de prudencia, puesto que las diferencias halladas pueden estar sesgadas por la diferencia muestral de estudiantes en cada subgrupo. En futuras investigaciones se deberían equiparar los $\mathrm{N}$ de cada una de las submuestras, y de esta manera lograr mayor homogeneidad en la representación de cada titulación.

Diferencias en Estrategias de Afrontamiento y Personalidad en función de la Edad.

Antes de realizar las pruebas estadísticas oportunas se establecieron 4 subgrupos según intervalos de edad: 17-22 años, 23-27 años, 28-32 años, 33 o más.

Tabla 4

ANOVA Estrategias de Afrontamiento y Personalidad en función de la Edad

\begin{tabular}{|c|c|c|c|c|c|c|}
\hline & & $\begin{array}{l}\text { Suma de } \\
\text { cuadrados }\end{array}$ & gl & $\begin{array}{c}\text { Media } \\
\text { cuadrática }\end{array}$ & $\mathrm{F}$ & Sig. \\
\hline \multirow{3}{*}{ Expresión Emocional Abierta } & Inter-grupos & 5,129 & 3 & 1,710 & 3,881 &, 009 \\
\hline & Intra-grupos & 194,723 & 442 & ,441 & & \\
\hline & Total & 199,852 & 445 & & & \\
\hline \multirow{3}{*}{ Evitación } & Inter-grupos & 8,643 & 3 & 2,881 & 5,108 &, 002 \\
\hline & Intra-grupos & 251,552 & 446 &, 564 & & \\
\hline & Total & 260,195 & 449 & & & \\
\hline \multirow{3}{*}{ FV Extraversión (-) } & Inter-grupos & 6,001 & 3 & 2,000 & 3,727 & 011 \\
\hline & Intra-grupos & 241,508 & 450 &, 537 & & \\
\hline & Total & 247,509 & 453 & & & \\
\hline
\end{tabular}


Al comparar los grupos de estudiantes según la edad aparecen diferencias en dos estrategias de Afrontamiento: Expresión Emocional Abierta y Evitación; así como en el Factor $V$ de Personalidad (Extraversión) (Tabla 4).

Para determinar los grupos de edad en las que se dan estas diferencias, se realiza la prueba de Scheffé. Se hace un análisis entre estos grupos comparando las medias (Tabla 5) cuyos resultados son comentados a continuación.

Las diferencias surgen entre los estudiantes más jóvenes (18-22 años) y los de mayor edad (33 años o más). Los primeros tienen puntuaciones más elevadas en, Expresión
Emocional Abierta y Evitación, mientras los de menor edad alcanzan una media de 2.66 en esta primera estrategia, y los de mayor edad de 2.02. Esto significa, que los más jóvenes utilizan más estrategias para afrontar el estrés centradas en la emoción. Respecto a la Evitación, los de menor edad tienen una media más elevada (3.15) en comparación con los de mayor edad (2.33), parece que los más jóvenes tienen un afrontamiento de tipo más afectivo ante las situaciones de estrés en comparación con los de mayor edad.

En cuanto a las características de personalidad, destaca la diferencia entre los estudiantes del grupo de 17 a 22 años y los de

Descriptivos Estrategias de Afrontamiento y Personalidad en función de la Edad

\begin{tabular}{|c|c|c|c|c|c|c|c|}
\hline & & & & & & \multicolumn{2}{|c|}{$\begin{array}{l}\text { Intervalo de confianza } \\
\text { para la media al } 95 \%\end{array}$} \\
\hline & & $\mathrm{N}$ & Media & $\begin{array}{l}\text { Desviación } \\
\text { típica }\end{array}$ & Error típico & $\begin{array}{l}\text { Límite } \\
\text { inferior }\end{array}$ & $\begin{array}{l}\text { Límite } \\
\text { superior }\end{array}$ \\
\hline \multirow{5}{*}{$\begin{array}{l}\text { Expresión } \\
\text { Emocional } \\
\text { Abierta }\end{array}$} & 1,00 & 370 & 2,66 & ,623 & ,032 & 2,60 & 2,73 \\
\hline & 2,00 & 58 & 2,56 & ,885 & 116 & 2,33 & 2,79 \\
\hline & 3,00 & 8 & 2,33 & ,766 & ,271 & 1,69 & 2,97 \\
\hline & 4,00 & 10 & 2,02 &, 530 & 167 & 1,64 & 2,40 \\
\hline & Total & 446 & 2,63 & 670 & ,032 & 2,57 & 2,69 \\
\hline \multirow{5}{*}{ Evitación } & 1,00 & 374 & 3,15 & ,753 & ,039 & 3,07 & 3,23 \\
\hline & 2,00 & 58 & 3,02 & ,716 & ,094 & 2,83 & 3,21 \\
\hline & 3,00 & 8 & 2,67 & ,840 & ,297 & 1,96 & 3,37 \\
\hline & 4,00 & 10 & 2,33 & 793 & ,251 & 1,77 & 2,90 \\
\hline & Total & 450 & 3,10 & ,761 & ,036 & 3,03 & 3,18 \\
\hline \multirow{5}{*}{$\begin{array}{c}\mathrm{FV} . \\
\text { Extraversión }\end{array}$} & 1,00 & 376 & 2,18 & 710 & ,037 & 2,11 & 2,25 \\
\hline & 2,00 & 60 & 2,51 & ,820 & 106 & 2,30 & 2,72 \\
\hline & 3,00 & 8 & 2,05 & ,510 & 180 & 1,62 & 2,48 \\
\hline & 4,00 & 10 & 2,12 & 1,104 & ,349 & 1,33 & 2,91 \\
\hline & Total & 454 & 2,22 & ,739 & ,035 & 2,15 & 2,29 \\
\hline
\end{tabular}


23 a 27años. Estos últimos se describen como más introvertidos, callados y solitarios (2.51) que los de edad inferior (2.18)

Diferencias en Resiliencia, Estrategias de Afrontamiento y Personalidad en función del Sexo.

El sexo aparece como la variable para la que más diferencias significativas se han encontrado. Estos datos han de ser interpretados sabiendo que la distribución entre hombres y mujeres, no es homogénea (aunque sí refleja la realidad en cuanto a la frecuencia de estudiantes de ambos sexos en las titulaciones estudiadas) como se reflejaba en la descripción de la muestra. (Tablas 6 y 7)

Encontramos diferencias significativas entre hombres y mujeres en dos de las subdimensiones de la Resiliencia: Satisfacción personal y Confianza en sí mismo, en ambas obtienen puntuaciones más altas las mujeres

Tabla 6

ANOVA Resiliencia, Bienestar Psicológico, Estrategias de Afrontamiento y Personalidad en función del Sexo

\begin{tabular}{|c|c|c|c|c|c|c|}
\hline & & $\begin{array}{l}\text { Suma de } \\
\text { cuadrados }\end{array}$ & gl & $\begin{array}{c}\text { Media } \\
\text { cuadrática }\end{array}$ & $\mathrm{F}$ & Sig. \\
\hline \multirow[t]{3}{*}{ Satisfacción } & Inter-grupos & 38,466 & 1 & 38,466 & 5,325 & 021 \\
\hline & Intra-grupos & 3286,799 & 455 & 7,224 & & \\
\hline & Total & 3325,265 & 456 & & & \\
\hline \multirow{3}{*}{ Confianza en sí mismo } & Inter-grupos & 193,264 & 1 & 193,264 & 15,486 & , 000 \\
\hline & Intra-grupos & 5628,541 & 451 & 12,480 & & \\
\hline & Total & 5821,806 & 452 & & & \\
\hline \multirow{3}{*}{ Crecimiento Personal } & Inter-grupos & 54,126 & 1 & 54,126 & 7,913 &, 005 \\
\hline & Intra-grupos & 3057,420 & 447 & 6,840 & & \\
\hline & Total & 3111,546 & 448 & & & \\
\hline \multirow[t]{3}{*}{ Propósito en la vida } & Inter-grupos & 198,165 & 1 & 198,165 & 15,730 & , 000 \\
\hline & Intra-grupos & 5681,663 & 451 & 12,598 & & \\
\hline & Total & 5879,828 & 452 & & & \\
\hline \multirow{3}{*}{ Evitación } & Inter-grupos & 3,231 & 1 & 3,231 & 5,635 & ,018 \\
\hline & Intra-grupos & 256,846 & 448 &, 573 & & \\
\hline & Total & 260,077 & 449 & & & \\
\hline \multirow{3}{*}{$\begin{array}{l}\text { Búsqueda de apoyo } \\
\text { social }\end{array}$} & Inter-grupos & 20,702 & 1 & 20,702 & 23,058 & ,000 \\
\hline & Intra-grupos & 397,730 & 443 & ,898 & & \\
\hline & Total & 418,432 & 444 & & & \\
\hline \multirow{3}{*}{ Fll Agradabilidad } & Inter-grupos & 3,294 & 1 & 3,294 & 22,399 & ,000 \\
\hline & Intra-grupos & 65,739 & 447 & , 147 & & \\
\hline & Total & 69,033 & 448 & & & \\
\hline \multirow[t]{3}{*}{ FIll Integridad } & Inter-grupos & 1,410 & 1 & 1,410 & 10,181 & ,002 \\
\hline & Intra-grupos & 62,305 & 450 & , 138 & & \\
\hline & Total & 63,715 & 451 & & & \\
\hline \multirow{3}{*}{ FIV Responsabilidad } & Inter-grupos & ,632 & 1 & ,632 & 5,306 & ,022 \\
\hline & Intra-grupos & 53,485 & 449 & , 119 & & \\
\hline & Total & 54,117 & 450 & & & \\
\hline \multirow[t]{3}{*}{ FV Extraversión } & Inter-grupos & 1,135 & 1 & 1,135 & 11,887 & ,001 \\
\hline & Intra-grupos & 42,871 & 449 & ,095 & & \\
\hline & Total & 44,005 & 450 & & & \\
\hline
\end{tabular}


Tabla 7

Descriptivos Resiliencia, Bienestar Psicológico, Estrategias de Afrontamiento y Personalidad en función del Sexo

\begin{tabular}{|c|c|c|c|c|c|}
\hline & & $\mathrm{N}$ & Media & Desviación típica & Error típico \\
\hline \multirow{3}{*}{ Satisfacción } & hombre & 101 & 14,83 & 3,124 & , 311 \\
\hline & mujer & 356 & 15,53 & 2,551 & , 135 \\
\hline & total & 457 & 15,38 & 2,700 & , 126 \\
\hline \multirow{3}{*}{$\begin{array}{l}\text { Confianza en sí } \\
\text { mismo }\end{array}$} & hombre & 100 & 26,36 & 4,071 & ,407 \\
\hline & mujer & 353 & 27,93 & 3,366 & 179 \\
\hline & total & 453 & 27,59 & 3,589 & , 169 \\
\hline \multirow{3}{*}{$\begin{array}{l}\text { Crecimiento } \\
\text { personal }\end{array}$} & hombre & 97 & 16,08 & 2,730 & ,277 \\
\hline & mujer & 352 & 16,93 & 2,583 & , 138 \\
\hline & total & 449 & 16,74 & 2,635 & 124 \\
\hline \multirow{3}{*}{ Propósito en la vida } & hombre & 101 & 17,89 & 4,005 & ,398 \\
\hline & mujer & 352 & 19,48 & 3,408 & , 182 \\
\hline & total & 453 & 19,13 & 3,607 & , 169 \\
\hline \multirow{3}{*}{ Evitación } & hombre & 100 & 2,95 & ,741 & ,074 \\
\hline & mujer & 350 & 3,15 & ,762 & 041 \\
\hline & total & 450 & 3,11 & ,761 & ,036 \\
\hline \multirow{3}{*}{$\begin{array}{l}\text { Búsqueda de } \\
\text { apoyo social }\end{array}$} & hombre & 99 & 3,37 & 1,077 & , 108 \\
\hline & mujer & 346 & 3,89 & ,907 & ,049 \\
\hline & total & 445 & 3,77 & 971 & ,046 \\
\hline \multirow{3}{*}{ Fll Agradabilidad } & hombre & 98 & 3,10 &, 425 & ,043 \\
\hline & mujer & 351 & 2,89 & ,371 & , 020 \\
\hline & total & 449 & 2,94 & ,393 & ,019 \\
\hline \multirow{3}{*}{ FIII Integridad } & hombre & 101 & 2,72 & ,442 &, 044 \\
\hline & mujer & 351 & 2,58 & ,349 & ,019 \\
\hline & total & 452 & 2,61 & ,376 & ,018 \\
\hline \multirow{3}{*}{$\begin{array}{c}\text { FIV } \\
\text { Responsabilidad }\end{array}$} & hombre & 100 & 2,98 & ,350 &, 035 \\
\hline & mujer & 351 & 2,89 & ,344 & ,018 \\
\hline & total & 451 & 2,91 & ,347 & ,016 \\
\hline \multirow{3}{*}{ FV Extraversión } & hombre & 100 & 3,14 &, 363 &, 036 \\
\hline & mujer & 351 & 3,02 & ,292 & ,016 \\
\hline & Total & 451 & 3,04 & ,313 & ,015 \\
\hline
\end{tabular}

que los hombres. En Satisfacción personal, las mujeres tienen una media de 15.53 mientras que en los hombres es de 14.83. Respecto a la Confianza en sí mismo, también las mujeres (27.93) se perciben con mayor capacidad que los hombres (26.36) para creer en ellas mismas.

Por otro lado, existen diferencias significativas en dos dimensiones del Bienestar psicológico: Crecimiento Personal y Propósito en la vida. En cuanto al Crecimiento Personal, la diferencia entre hombres (16.08) y mujeres (16.93) es mínima, aunque significativa. Sin embargo, donde sí se encuentran valores más distanciados es en Propósito en la vida, donde las mujeres (19.48) obtienen puntuaciones más altas respecto con los hombres (17.89). Las mujeres parecen tener más claro y definido el significado y sentido de su vida. 
También se observan diferencias en dos de las estrategias de afrontamiento empleadas ante situaciones de estrés. Las mujeres (3.15) puntúan más alto que los hombres (2.95) en Evitación y también en Búsqueda de apoyo social, para la cual las mujeres obtienen una media de 3.89 frente a los hombres de 3.37. Parece que las mujeres ante situaciones de estrés emplean más estrategias de afrontamiento de tipo afectivo que los hombres.

Finalmente, indicar las diferencias resultantes entre la valoración que hacen los hombres desus características de personalidad y las que hacen las mujeres. Para los tres factores que han resultado significativos, los hombres tienen puntuaciones más elevadas que las mujeres. En Agradabilidad, los hombres (3.10) se describen más tolerantes, pacíficos y serenos que las mujeres (2.89). En cuanto a Integridad los hombres $(2,72)$ se perciben más profundos, reflexivos y receptivos que las mujeres (2.58). En la misma línea de respuesta que los anteriores, los hombres (2.98) obtienen puntuaciones ligeramente más elevadas que las mujeres (2.89) en Responsabilidad e igualmente en Extraversión.

Diferencias en Personalidad en función de la presencia o no de enfermedad.

Respecto a la existencia de diferencias entre el grupo de enfermos y no enfermos en las distintas escalas, los hallazgos encontrados son escasos y difícilmente interpretables por ello, de tal manera que sólo ha resultado significativa para el Factor de Personalidad Extraversión, para un nivel de significación de $p<0.05$ (.013). Al comparar sus medias se observa una puntuación mínimamente más elevada entre los estudiantes que padecen una enfermedad (3.12) y aquellos que no la tienen (3.03). Los primeros se describen con mayores niveles de extraversión, más sociables, alegres, abiertos y habladores, frente a los segundos.

\section{DISCUSIÓN}

Teniendo en cuenta los resultados anteriormente expuestos, se puede llegar a las siguientes conclusiones:

Existe una clara red de interrelación entre la Resiliencia, las dimensiones del Bienestar psicológico, las Estrategias de Afrontamiento y las características de Personalidad. Se confirman los hallazgos de investigaciones previas donde se demuestra la estrecha relación que existe entre estos constructos psicológicos, responsables del funcionamiento óptimo de la persona (Friborg, Barlaug, Martinussen, RosenvingeyHjemdal, 2005; Kobasa, 1979; Vázquez, Hervás, Rahonay Gómez, 2009)

Principalmente se destacan la relación entre las dimensiones de la Resiliencia y las del Bienestar psicológico, así como la relación de éstas con dos de las Estrategias de Afrontamiento: Focalización en la solución de problemas y Reevaluación positiva. Respecto a la primera afirmación, se podría interpretar que al aumentar los niveles de las distintas subdimensiones de la Resiliencia, aumentan las dimensiones del Bienestar psicológico, sin poder determinar ningún tipo de relación causal. Por otro lado, estos resultados confirman uno de los planteamientos inciales de este estudio, el cual intuía que las personas resilientes, así como aquellas que gozan de un elevado estado de bienestar, emplean estrategias de afrontamiento específicas, en este caso, la Focalización en la solución de problemas y la Reevaluación positiva. En cuanto a la dimensión de personalidad que ha resultado más implicada en todos los análisis ha sido la Estabilidad Emocional, parece entenderse que esta gran dimensión de la personalidad asume un alto grado de responsabilidad en el afrontamiento de la adversidad así como en el logro del bienestar personal.

Finalmente, al comparar los distintos grupos de la muestra, vemos que existen diferencias en algunas dimensiones de la Resiliencia y del Bienestar psicológico, así como en las Estrategias de Afrontamiento 
y variables de Personalidad, cuando se atiende a la Titulación, Edad y Sexo; pero sin embargo la presencia de enfermedad no arroja ningún tipo de información relevante al respecto. Se entiende, que quizás para dar una respuesta de mayor solidez científica a esta última cuestión, debería aumentarse el $N$ de la submuestra de estudiantes con algún tipo de patología crónica diagnosticada. En éste sentido, ésta podría ser entendida como una de las principales limitaciones del estudio, impidiendo extraer conclusiones, principalmente en lo que se refiere a la Resiliencia y a las Estrategias de Afrontamiento, donde la literatura científica reconoce la presencia de enfermedad como un hecho relevante que hace a la persona más resiliente y con más estrategias de afrontamiento al estrés (Broadbent, Petrie, Main, y Weinman, 2006; Lazarus y Folkman, 1986). Cómo ha sido comentado en el apartado de los resultados, esta limitación no sólo se encuentra en esta útlima variable comentada, sino que también se ven implicadas otras como la edad, el sexo y la titulación, en las que se deberían logar representaciones mayores y más homogéneas.

Para finalizar, destacar la importancia de continuar avanzando en esta línea de investigación que pretende aproximarse cada vez más al conocimiento de los factores que intervienen en el desarrollo óptimo de la persona, contribuyendo de este modo al objetivo común sobre el que actualmente se centra gran parte de los estudios psicológicos (Vázquez y Hervás, 2009). Conocer los componentes de la Resiliencia y el Bienestar psicológico, así como las Estrategias de Afrontamiento para hacer frente al estrés, permite establecer y desarrollar líneas de intervención psicológica, individuales y colectivas, que favorezcan un mayor estado de bienestar personal con todas las repercusiones que ello conlleva, tanto para la persona como para la propia sociedad.

\section{- Conflicto de intereses}

Los autores declaran no tener ningún conflicto de intereses.

\section{REFERENCIAS}

Becoña, E. (2006). Resiliencia: definición, características y utilidad del concepto. Revista de Psicopatología y Psicología Clínica, 11 (3), 125-146. DOI: 10.5944/ rppc.vol. 1 1.num.3.2006.4024

Benard, B. (2004). Resilience y What we have learned. San Francisco: West Ed.

Broadbent, E., Petrie, K. J., Main, J., y Weinman, J. (2006). The Brief Illness Perception Questionnaire (BIPQ). Journal of Psychosomatic Research, 60, 631-637. DOI: 10.1016/i.jpsychores.2005.10.020

Büssing, A., Ostermann, T., y Koenig, H.G. (2007). Relevance of religion and spirituality in german patients with chronic diseases. International Journal Psychiatry in Medicine, 37, 39-57. DOI: 10.2190/60W7-16612623-6042

Díaz, D., Rodríguez-Carvajal, R., Blanco, A., Moreno-Jiménez, B., Gallardo, I., Valle, C., y van Dierendonck, D. (2006). Adaptación española de las escalas de bienestar psicológico de Ryff. Psichotema, 18 (3), 572-577.

Friborg, O., Barlaug, D., Martinussen, M., Rosenvinge, J.H., yHjemdal, O. (2005). Resilience in relation to personality and intelligence. International Journal of Methods in Psychiatric Research, 14, 29-40. DOI: 10.1002/mpr. 15

Goldberg, L.R. (1981). Language and individual differences: the search for universals in personality lexicons. Review of Personality and Social Psychology, 2, 141-165.

Heilemann, M., Lee, K.,yKury, F.S. (2003). Psychometric properties of the Spanish version of the Resilience Scale. Journal Nurs Meas, 11 (1), 61-72. DOI: $10.1891 / 106137403780954976$

Iraegui, A.,y Quevedo-Aguado, M.P. (2002). Aproximación psicolingüística al estudio de la personalidad en español: Una propuesta taxonómica. Iberpsicología: Revista Electrónica de la Federación española de Asociaciones de Psicología, Vol. 7, No. 1.

Kobasa, S.C. (1979): Personality and resistance 
to illness. American Journal of Community Psychology, 7, 413-423. DOI: 10.1007/ BF00894383

Lazarus, R.S.,y Folkman, S. (1986). Estrés y procesos cognitivos: Martínez Roca.

Luthar, S. (2006). Resilience in development: A synthesis of researach across five decaces. En D. Cicchetti y D.J. Cohen (Eds.), Developmental psychopathology: Risk, disorder, and adaptation, Vol. 3 (2th edition). Nueva York: Wiley. DOI: 10.1002/9780470939406.ch20

Rutter, M. (1985). Resilience in the face of adversity: protective factors and resistance to psychiatric disorder. British Journal of Psychiatry, 147, 598-611. DOI: 10.1192/ bip. 147.6 .598

Rutter, M. (1987). Psychosocial resilience and protective mechanisms. American Journal of Orthopsychiatry, 57, 316-331. http:// dx.doi.org/10.1111/i.1939-0025.1987. tb03541.x

Rutter, M. (2007). Resilience, competence and coping. Child Abuse and Neglect, 31, 205 209. DOI: 10.1016/i.chiabu.2007.02.001
Ryff, C. (1989). Happiness in everythin, or is it? Explorations on the meaning of psychological well-being. Journal of Personality and Social Psychology, 57, 1069-81. https://doi. org/10.1037/0022-3514.57.6.1069

Sandin, B., y Chorot, P. (2003).Cuestionario del Afrontamiento del estrés (CAE): Desarrollo y situación preliminar. Revista de Psicopatología y Psicología Clínica, 8, 39-54. DOI: 10.5944/rppc.vol.12. num.3.2007.4044

Vázquez, C., y Hervás, G. (2009). La ciencia del bienestar. Fundamentos de una psicología positiva. Madrid: Alianza Editorial.

Vázquez, C., Hervás, G., Rahona, J. J., y Gómez, D. (2009). Bienestar psicológico y salud: Aportaciones desde la Psicología. Psicología Clínica y de la Salud, 5, 15-28.

Wagnild, G.M., y Young, H.M. (1993). Development and psychometric evaluation of the resilience scale. Journal of Nursing Measurement, 1, 165-178.

Wolin, S. J.,y Wolin, S. (1993). The resilient self: how survivors of troubled families rise above adversity. Nueva York: VillardBooks. 